OPEN ACCESS

Edited by:

Gianluca Franceschini, Catholic University of the Sacred Heart, Italy

Reviewed by:

Charles M. Malata,

Anglia Ruskin University,

United Kingdom

Marzia Salgarello,

Catholic University of the Sacred

Heart, Italy

Lorenzo Scardina,

A. Gemelli University Hospital

Foundation, Italy

Antonio Toesca,

European Institute of Oncology (IEO),

Italy

${ }^{*}$ Correspondence:

Hyung Seok Park

hyungseokpark.md@gmail.com; imgenius@yuhs.ac

Specialty section: This article was submitted to Surgical Oncology,

a section of the journal

Frontiers in Oncology

Received: 13 August 2020 Accepted: 19 November 2020 Published: 08 January 2021

Citation:

Lee J, Park HS, Lee H, Lee DW, Song SY, Lew DH, Kim JY, Park S and Kim SI (2021) Post-Operative Complications and Nipple Necrosis

Rates Between Conventional and Robotic Nipple-Sparing Mastectomy.

Front. Oncol. 10:594388.

doi: 10.3389/fonc.2020.594388

\section{Post-Operative Complications and Nipple Necrosis Rates Between Conventional and Robotic Nipple-Sparing Mastectomy}

\author{
Jeea Lee ${ }^{1}$, Hyung Seok Park ${ }^{1 *}$, Haemin Lee ${ }^{1}$, Dong Won Lee ${ }^{2}$, Seung Yong Song ${ }^{2}$, \\ Dae Hyun Lew ${ }^{2}$, Jee Ye Kim ${ }^{1}$, Seho Park ${ }^{1}$ and Seung II Kim ${ }^{1}$

\begin{abstract}
1 Department of Surgery, Yonsei University College of Medicine, Seoul, South Korea, ${ }^{2}$ Department of Plastic and
\end{abstract} \\ Reconstructive Surgery, Yonsei University College of Medicine, Seoul, South Korea
}

Purpose: This study is to directly compare surgical outcomes between conventional nipple-sparing mastectomy (CNSM) and robot-assisted nipple-sparing mastectomy (RNSM).

Materials and Method: For this case-control study, 369 cases of 333 patients who underwent CNSM or RNSM with immediate reconstruction between November 2016 and January 2019 at Severance Hospital in Seoul, Republic of Korea were reviewed. Patients with stage IV breast cancer $(n=1)$, receiving neoadjuvant chemotherapy $(n=43)$, or subjected to previous operations $(n=14)$ or radiotherapy on the breasts were excluded. The main outcomes were comparing rates of post-operative complications, of high-grade post-operative complications as defined by the Clavien-Dindo classification, and nipple necrosis between the CNSM and the RNSM groups.

Results: A total of 311 cases, including 270 CNSMs and 41 RNSMs, were analyzed. The rates of post-operative nipple necrosis ( $p=0.026,2.4 \mathrm{vs} .15 .2 \%$ ) and of high-grade postoperative complications ( $p=0.031,34.8 v s .17 .1 \%)$ in the RNSM group were significantly lower than those in the CNSM group.

Conclusion: RNSM was associated with lower rates of high-grade post-operative complications and nipple necrosis than CNSM for patients with small breast volumes and less ptotic breasts.

Keywords: breast neoplasms, robotic mastectomy, nipple-sparing mastectomy, minimal invasive surgery, nipple necrosis

\section{INTRODUCTION}

Nipple-sparing mastectomy (NSM) has been widely applied to women with early breast cancer or BRCA 1/2 mutations (1-4). Because NSM preserves the nipple areolar complex (NAC) and overlying skin, NSM results in better cosmetic outcomes coupled with oncologic safety for those patients, compared to conventional total mastectomy or skin-sparing mastectomy (4-9). 
Nipple necrosis is one of the most common complications after NSM $(1,2,10,11)$. Previous studies reported $0-48 \%$ of nipple ischemia or nipple necrosis in patients undergoing NSM with immediate reconstruction $(1,12)$. In order to reduce nipple ischemia or necrosis, various techniques have been proposed in previous studies $(12,13)$. Rusby et al. showed that placement of incisions far from the NAC and reconstruction using a tissue expander (T/E) reduced the risk of NAC necrosis (12). Petit et al. reported that leaving a layer $5 \mathrm{~mm}$ of glandular tissue beneath the NAC for preserving its blood supply is beneficial to reduce NAC necrosis (13). However, there is no universal solution for reducing nipple necrosis after NSM.

Many surgeons have tried to develop various incisions in NSM to deliver better cosmetic outcomes (14-16). Robotassisted nipple-sparing mastectomy (RNSM) is a procedure that uses robotic systems through axillary or lateral incisions, which results in no scars in the overlying skin. A previous study reported that RNSM presented with low rates of nipple necrosis (17-19). However, there has been, to our knowledge, a lack of comparisons between RNSM and conventional NSM (CNSM) in terms of nipple necrosis rates.

This study aimed to evaluate nipple necrosis rates between RNSM and CNSM. Additionally, grades and rates of complications after the two procedures were analyzed and compared.

\section{MATERIALS AND METHODS}

\section{Patients}

A total of 333 patients in the present study had undergone CNSM or RNSM between November 2016 and January 2019 at Severance Hospital, Seoul, Korea. Their medical records and post-operative photographs taken by plastic surgeons were retrospectively reviewed. The photographs were taken on 1, 2, 3, 5, 7, and 9 days after the operation of autologous reconstruction routinely. After a prosthetic reconstruction, post-operative photographs were taken on $1,2,4,6$, and 8 days after the operation. In an outpatient department, plastic surgeons take the photographs as needed. Exclusion criteria were the presence of stage IV disease $(n=1)$, treatment with neoadjuvant chemotherapy $(\mathrm{n}=43)$, and previous operation or radiation history $(\mathrm{n}=14)$. This resulted in a total of 311 cases, 270 cases with CNSM and 41 cases with RNSM, from 275 patients being enrolled in the study (Figure 1). Among them, 36 patients underwent either bilateral CNSM or RNSM. There was no male patient in this study because patients who underwent immediate reconstruction after mastectomy were collected.

Clinicopathologic features, including age, BMI, breast volume, ptosis, disease entities, TNM stage, estrogen and progesterone receptor, human epidermal growth factor receptor (HER) 2 status, Ki 67 levels, adjuvant therapies, reconstruction methods, duration of hospital stays, and operation times were analyzed. Post-operative complications through 1-28 months, including nipple ischemia or necrosis, skin ischemia or necrosis, infection, bleeding, lymphedema, limitation of shoulder movement, contracture, seroma, wound dehiscence, and arterial thrombus, were also analyzed. Nipple ischemia in this study was defined as a clinical ischemic color change in the NAC. Nipple necrosis was defined as full-thickness necrosis of the NAC requiring surgical intervention (1). Grades of post-operative complications were analyzed according to the Clavien-Dindo classification (20).

\section{Procedures}

CNSM was performed using various methods by three breast surgeons (Figure 2). Immediate reconstruction, including tissue expander (T/E), direct-to-implant (DTI), Latissimus dorsi (LD) flap, and transverse rectus abdominis musculocutaneous (TRAM) flap, was performed according to surgeons' and patients' preferences by three plastic surgeons. A deep inferior epigastric perforator flap was included in the TRAM flap. RNSM was performed via single axillary or lateral incision by a breast surgeon. Gas or gasless technique in robotic mastectomy was

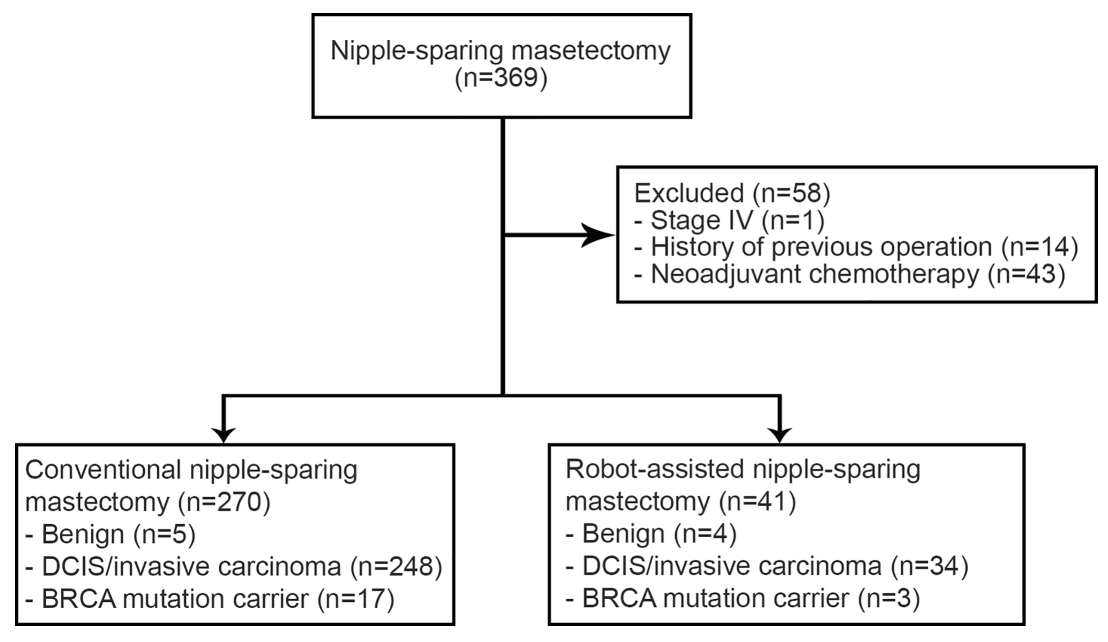

FIGURE 1 | Schematic diagram of the study population. 
A

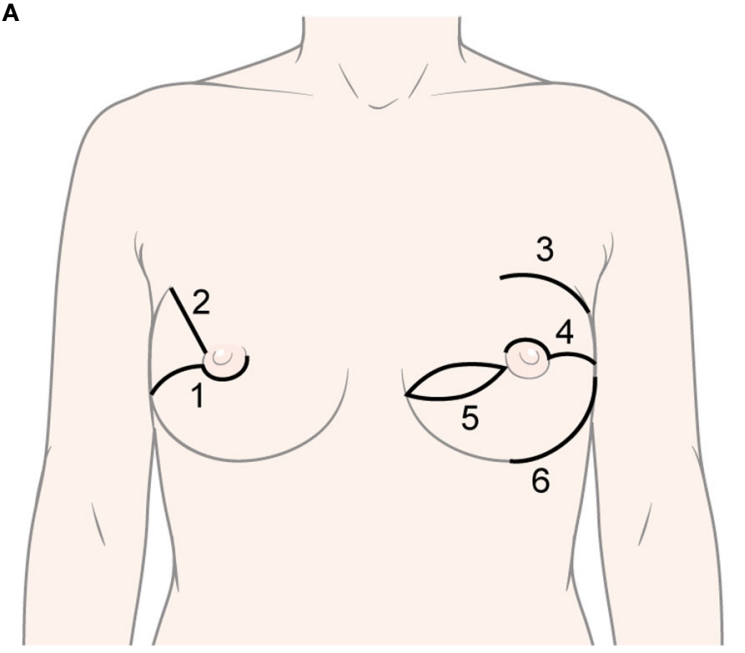

B

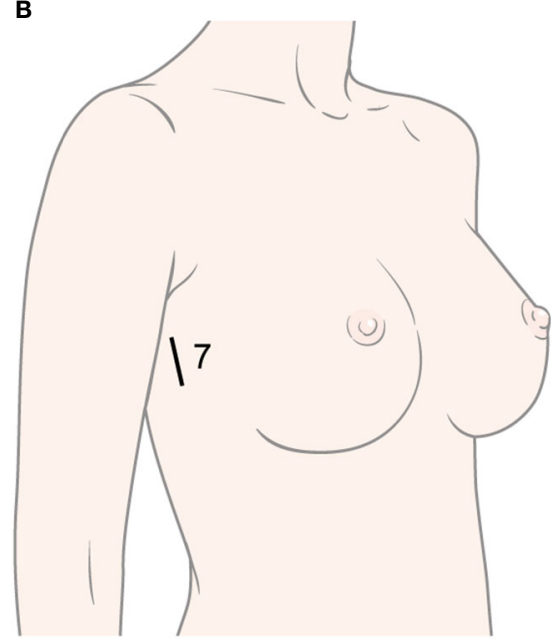

FIGURE 2 | Various types of incisions in conventional nipple-sparing mastectomy and robot-assisted nipple-sparing mastectomy. (A) Types of incision in conventional nipple-sparing mastectomy: 1) Lower-periareolar incision with/without extension, 2) Radial incision, 3) Curvilinear incision, 4) Upper-periareolar incision with/without extension, 5) Elliptical incision, 6) Inframammary fold incision; (B) Type of incision in robot-assisted nipple-sparing mastectomy: 7) Lateral incision.

applied to patients with early breast cancer or BRCA mutations $(17-19,21)$. The detailed techniques were described in previous studies $(17-19,21)$. T/E insertion or DTI was applied for immediate reconstruction in those patients $(19,21)$.

\section{Pathologic Evaluations}

Estrogen receptor (ER), progesterone receptor (PR), HER2 status, and Ki 67 levels were analyzed by immunohistochemistry (IHC), as described in previous studies (22). In brief, positivity for ER and PR was defined as $\geq 1 \%$ nuclear staining in IHC. HER $22+$ in IHC and amplification in fluorescence in situ hybridization/silver in situ hybridization or $3+$ in IHC were considered overexpression according to ASCO/CAP guidelines (23). The cut-off values for Ki 67 staining for low and high proliferative index were $<$ and $\geq 14 \%$ staining in IHC, respectively (24). TNM stage was classified according to anatomic stage as in the AJCC $8^{\text {th }}$ edition. Nipple margins were reviewed from both intra-operative frozen and postoperative permanent pathologic evaluations.

\section{Adjuvant Therapies}

Chemotherapy, endocrine therapy, and radiation therapy were delivered according to standard guidelines or physicians' preferences (25). Patients with HER2-positive disease and tumor sizes $\geq 1 \mathrm{~cm}$ routinely received adjuvant trastuzumab therapy.

\section{Statistics}

A learning curve of RNSM for total operation time was analyzed using three-day moving average curves (3D-MAC), and the cumulative sum (CUSUM) technique. 3D-MAC is used to analyze the existence of a learning curve (26). This simple moving average is defined as the mean value of previous 3 days data points (27). The CUSUM technique is a statistical method to assess the learning curve quantitatively and to calculate the sequential difference between the individual and the mean value of all data (28). The CUSUM is estimated by CUSUM $=\sum_{i=1}^{n}(x i-\mu)$, where $x i$ is an individual operation time, and $\mu$ is the mean value of overall operation time (29).

Categorical variables were analyzed using either Chi-square test or Fisher's exact test, if indicated. Continuous variables were analyzed using either Student's t test or Mann-Whitney test, if indicated. All tests were two-sided. Multivariate analysis was performed using binary regression with backward elimination (conditional) to evaluate risk factors related with high-grade complications (Clavien-Dindo classification $\geq$ grade III). A $p$-value less than 0.05 was considered to be statistically significant. All statistical analyses were performed using the SPSS software, version 25 (SPSS Inc., Chicago, IL). We did not use a statistical matching technique due to the limited sample size. Missing values were imputed as null values.

\section{Ethics}

This study was approved by the institutional review board at Severance Hospital (4-2019-0510).

\section{RESULTS}

The clinicopathologic features of the enrolled patients are shown in Table 1. The mean age of patients was $45.93 \pm 8.34$ (data not shown). There were no differences in clinicopathologic features between the CNSM and RNSM groups, except in breast volumes, laterality, and ptosis. Ptotic breasts were more frequent and breast volumes were larger in the CNSM group. Others subgroup in BRCA mutation included three cases with PALB2 mutations (Table 1).

Post-operative outcomes, including length of hospital stay and operation times are shown in Table 2. The length of hospital stay in 
TABLE 1 | Clinicopathologic characteristics of the study population.

\begin{tabular}{|c|c|c|c|c|}
\hline & & $\begin{array}{c}\text { CNSM } \\
(n=270)\end{array}$ & $\begin{array}{l}\text { RNSM } \\
(n=41)\end{array}$ & $p$-value ${ }^{b}$ \\
\hline Age (years) & & $46 \pm 8.0$ & $44 \pm 10.0$ & $0.075^{c}$ \\
\hline BMl $\left(\mathrm{kg} / \mathrm{m}^{2}\right)$ & & $22.5 \pm 3.1$ & $21.7 \pm 2.3$ & $0.065^{c}$ \\
\hline Breast volume (g) & & $428 \pm 222.0$ & $326 \pm 143.0$ & $0.002^{c}$ \\
\hline \multirow[t]{2}{*}{ Laterality } & Unilateral & $216(80.0)$ & $23(56.1)$ & 0.001 \\
\hline & Bilateral & $54(20)$ & $18(43.9)$ & \\
\hline \multirow[t]{5}{*}{ Ptosis } & Normal & $136(50.4)$ & $32(78.0)$ & 0.004 \\
\hline & Mild & $56(20.7)$ & 8 (19.5) & \\
\hline & Moderate & 36 (13.3) & $0(0.0)$ & \\
\hline & Severe & $38(14.1)$ & $1(2.4)$ & \\
\hline & Pseudoptosis & $2(0.7)$ & $0(0.0)$ & \\
\hline \multirow[t]{4}{*}{ BRCA1 mutation } & No & $89(81.7)$ & $16(94.1)$ & 0.913 \\
\hline & Yes & $11(10.1)$ & $1(5.9)$ & \\
\hline & VOUS & $6(5.5)$ & $0(0.0)$ & \\
\hline & Others & $3(2.8)$ & $0(0.0)$ & \\
\hline \multirow[t]{4}{*}{ BRCA2 mutation } & No & $89(81.7)$ & $10(58.8)$ & 0.050 \\
\hline & Yes & $12(11.0)$ & $6(35.5)$ & \\
\hline & VOUS & $5(4.6)$ & $1(5.9)$ & \\
\hline & Others & $3(2.8)$ & $0(0.0)$ & \\
\hline \multirow[t]{4}{*}{ Diagnosis } & Benign & $5(1.9)$ & $4(9.8)$ & 0.069 \\
\hline & DCIS & $63(23.3)$ & $9(22.0)$ & \\
\hline & Invasive carcinoma & $185(68.5)$ & $25(61.0)$ & \\
\hline & BRCA mutation carrier & $17(6.3)$ & $3(7.3)$ & \\
\hline \multirow[t]{2}{*}{$E R^{a}$} & Negative & $49(19.8)$ & $3(8.8)$ & 0.123 \\
\hline & Positive & $199(80.2)$ & $31(91.2)$ & \\
\hline \multirow[t]{2}{*}{$P R^{a}$} & Negative & $64(25.8)$ & $8(23.5)$ & 0.775 \\
\hline & Positive & $184(74.2)$ & $26(76.5)$ & \\
\hline \multirow[t]{2}{*}{$\mathrm{HER}^{\mathrm{a}}{ }^{\mathrm{a}}$} & Negative & $174(76.3)$ & $21(63.6)$ & 0.117 \\
\hline & Positive & $54(23.7)$ & $12(36.4)$ & \\
\hline \multirow[t]{2}{*}{$\mathrm{Ki} 67^{\mathrm{a}}$} & Low (<14\%) & $108(44.3)$ & 13 (38.2) & 0.632 \\
\hline & High ( $\geq 14 \%)$ & $136(55.7)$ & $21(61.8)$ & \\
\hline \multirow[t]{3}{*}{ Histologic grade ${ }^{a}$} & Grade I & $59(23.8)$ & $5(14.7)$ & 0.445 \\
\hline & Grade ॥ & $144(58.1)$ & $21(61.8)$ & \\
\hline & Grade III & $45(18.1)$ & $8(23.5)$ & \\
\hline \multirow[t]{3}{*}{$\mathrm{T}^{\mathrm{a}}$} & Tis & 67 (27.0) & $11(32.4)$ & 0.615 \\
\hline & $\mathrm{T} 1$ & $144(58.1)$ & $20(58.8)$ & \\
\hline & $\mathrm{T} 2$ & $37(14.9)$ & $3(8.8)$ & \\
\hline \multirow[t]{4}{*}{$\mathrm{N}^{\mathrm{a}}$} & NO & $210(86.1)$ & 30 (88.2) & 0.653 \\
\hline & N1 & 29 (11.9) & $3(8.8)$ & \\
\hline & $\mathrm{N} 2$ & $4(1.6)$ & $1(2.9)$ & \\
\hline & N3 & $1(0.4)$ & $0(0.0)$ & \\
\hline \multirow[t]{4}{*}{ TNM stage $^{a}$} & 0 & $68(27.4)$ & $8(23.5)$ & 0.766 \\
\hline & I & $126(50.8)$ & $20(58.8)$ & \\
\hline & $\|$ & $48(19.4)$ & $5(14.7)$ & \\
\hline & III & $6(2.4)$ & $1(2.9)$ & \\
\hline \multirow[t]{2}{*}{ Adjuvant chemotherapy ${ }^{\mathrm{a}}$} & No & 167 (67.3) & $23(67.6)$ & 0.971 \\
\hline & Yes & $81(32.7)$ & $11(32.4)$ & \\
\hline \multirow[t]{2}{*}{ Radiotherapy ${ }^{\mathrm{a}}$} & No & $220(88.7)$ & $30(88.2)$ & 0.935 \\
\hline & Yes & $28(11.3)$ & $4(11.8)$ & \\
\hline \multirow[t]{2}{*}{ Hormone therapy ${ }^{\mathrm{a}}$} & No & $58(23.4)$ & $5(14.7)$ & 0.254 \\
\hline & Yes & $190(76.6)$ & $29(85.3)$ & \\
\hline \multirow[t]{2}{*}{ Target therapy ${ }^{a}$} & No & $231(93.1)$ & $29(85.3)$ & 0.161 \\
\hline & Yes & 17 (6.9) & 5 (14.7) & \\
\hline \multirow[t]{2}{*}{ Recurrence $^{a}$} & No & $246(99.2)$ & $41(100.0)$ & $>0.999$ \\
\hline & Yes & $2(0.8)$ & $0(0.0)$ & \\
\hline
\end{tabular}

Values are represented as mean $\pm S D$ or number (percentage).

BMI, body mass index; CNSM, conventional nipple-sparing mastectomy; DCIS, ductal carcinoma in situ, ER, estrogen receptor; HER, human epidermal growth factor receptor;

$P R$, progesterone receptor; RNSM, robot-assisted nipple-sparing mastectomy; VOUS, variants of unknown significance.

${ }^{2} 29$ cases of benign disease or BRCA mutation carriers were not included $(n=282)$.

${ }^{b}$ Chi-square test or Fisher's exact test.

'Student's t test or Mann-Whitney test. 
TABLE 2 | Surgical methods and post-operative outcomes.

\begin{tabular}{|c|c|c|c|c|}
\hline & & $\begin{array}{c}\text { CNSM } \\
(n=270)\end{array}$ & $\begin{array}{l}\text { RNSM } \\
(n=41)\end{array}$ & $p$-value ${ }^{b}$ \\
\hline Hospital stay (days) & & $12 \pm 3$ & $14 \pm 4$ & $0.001^{c}$ \\
\hline Total operation time (min) & & $303.9 \pm 195.9$ & $308.9 \pm 75.5$ & $<0.001^{\mathrm{c}}$ \\
\hline Mastectomy time (min) & & $104.5 \pm 40.5$ & $181.5 \pm 44.7$ & $<0.001^{\mathrm{c}}$ \\
\hline Console time (min) & & - & $64 \pm 40$ & - \\
\hline Reconstruction time (min) & & $196.8 \pm 182.5$ & $140.5 \pm 52.5$ & $0.019^{c}$ \\
\hline \multirow[t]{2}{*}{ Operation site } & Left & $139(51.5)$ & $19(46.3)$ & 0.616 \\
\hline & Right & $131(48.5)$ & $22(53.7)$ & \\
\hline \multirow[t]{4}{*}{ Reconstruction types } & $\mathrm{T} / \mathrm{E}$ & $190(70.4)$ & $21(51.2)$ & $<0.001$ \\
\hline & DTI & $5(1.9)$ & $20(48.8)$ & \\
\hline & TRAM & $73(27.0)$ & $0(0.0)$ & \\
\hline & LD & $2(0.7)$ & $0(0.0)$ & \\
\hline \multirow[t]{7}{*}{ Incision types } & IMF & $51(18.9)$ & $0(0.0)$ & $<0.001$ \\
\hline & Radial & $32(11.9)$ & $0(0.0)$ & \\
\hline & Upper-periareolar with extension & $120(44.4)$ & $0(0.0)$ & \\
\hline & Lower-periareolar with extension & $52(19.3)$ & $0(0.0)$ & \\
\hline & Circumareolar & $3(1.1)$ & $0(0.0)$ & \\
\hline & Elliptical & $12(4.4)$ & $0(0.0)$ & \\
\hline & Lateral or axillary & $0(0.0)$ & $41(100.0)$ & \\
\hline \multirow[t]{2}{*}{ SLNB ${ }^{a}$} & No & $20(7.7)$ & $2(5.9)$ & $>0.99$ \\
\hline & Yes & 239 (92.3) & $32(94.1)$ & \\
\hline \multirow[t]{2}{*}{$\mathrm{ALND}^{\mathrm{a}}$} & No & $224(86.5)$ & 31 (91.2) & 0.592 \\
\hline & Yes & $35(13.5)$ & $3(8.8)$ & \\
\hline \multirow[t]{2}{*}{ Margin status $^{a}$} & No & $240(96.8)$ & $33(97.1)$ & 0.423 \\
\hline & Yes & $3(1.2)$ & $1(2.9)$ & \\
\hline
\end{tabular}

Values are represented as mean $\pm S D$ or number (percentage).

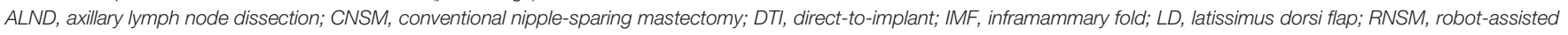
nipple-sparing mastectomy; SLNB, sentinel lymph node biopsy; T/E, tissue expander; TRAM, transverse rectus abdominis musculocutaneous flap.

${ }^{2} 29$ cases of benign disease or BRCA mutation carriers were not included $(n=282)$.

${ }^{b}$ Chi-square test or Fisher's exact test.

'Student's t test or Mann-Whitney test.

the RNSM group was greater than in the CNSM group $(p<0.001$, $14 \pm 4$ vs. $12 \pm 3$ days), and the same held for total operation time $(p<0.001,308.9 \pm 75.5$ vs. $303.9 \pm 195.9 \mathrm{~min})$. Mastectomy time was longer in the RNSM group than the CNSM group $(p<0.001$, $181.5 \pm 44.7$ vs. $104.5 \pm 40.5 \mathrm{~min})$, Reconstruction time was longer in the CNSM group than the RNSM group $(p=0.019,196.8 \pm 182.5$ vs. $140.5 \pm 52.5 \mathrm{~min})$.

$\mathrm{T} / \mathrm{E}$ was the most common method for immediate reconstruction in both groups (Table 2). TRAM is the second most common method for immediate reconstruction in the CNSM group. Approximately half of the patients underwent DTI after RNSM.

Incision types are described in Table 2. Periareolar with extension was the most common incision in the CNSM group, followed by IMF, radial, elliptical, and curvilinear incision. Lateral or axillary incision was only used in the RNSM group. Incision types between the two groups were significantly different $(p<0.001)$. There was no significant difference of margin status between two groups. The CNSM group included one nipple and two superficial margins of tumor involvement. The RNSM group had one superficial margin involvement of tumor (Table 2). One patient who underwent RNSM showed false negative in subareolar mass in frozen section. Because the final pathology revealed invasive ductal carcinoma in the mass, NAC was sacrificed.

Figure 3 shows grades of post-operative complications and nipple necrosis rates between the two groups. Post-operative complication rates were not different between the CNSM and RNSM groups $(p=0.176,58.5$ vs. $46.3 \%)$. There was no significant difference in implant loss and infection rates between the groups (for implant loss, $p=0.347,0.7 \%$ for the CNSM group vs. $2.4 \%$ for the RNSM group, for infection, $p=$ $0.101,2.2 \%$ for the CNSM group vs. $7.3 \%$ for the RNSM group, data not shown). Post-operative complications requiring surgical intervention, such as wound revision, drain re-insertion, fat graft injection for volume defects, and implant removal were more common in the CNSM group ( $p=0.031$, grade $\geq \mathrm{III}, 34.8 \%$ vs. $17.1 \%)$. Nipple necrosis rate was significantly lower in the RNSM group than in the CNSM group ( $p=0.026,2.4$ vs. $15.2 \%$ ).

Multivariate analysis was conducted to evaluate risk factors related to high-grade complications. The rate of high-grade complications (grade $\geq$ III) was statistically associated with the methods of the mastectomy and the operation time ( $p=0.046$ and $p<0.001$ ) (Table 3).

\section{DISCUSSION}

Our study demonstrated the advantage of RNSM compared to the CNSM in terms of nipple necrosis rate. Previous studies suggested that certain incision types are significantly associated with nipple necrosis because the viability of the NAC is mainly maintained by blood supply from dermal layers $(4,30)$. Another 
A

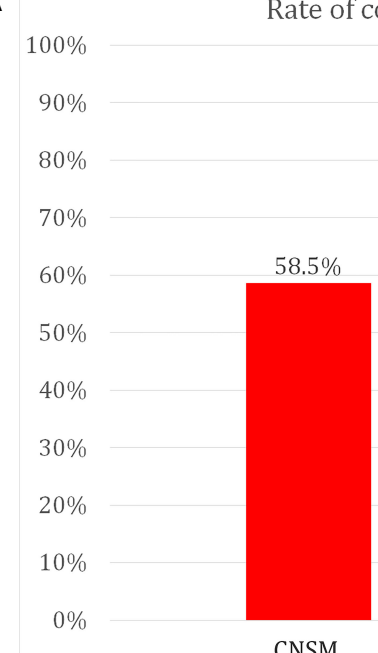

B

Grade of complications $(p=0.031)$
176)

$46.3 \%$

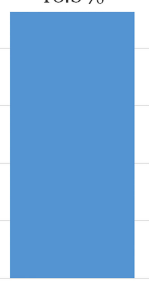

RNSM

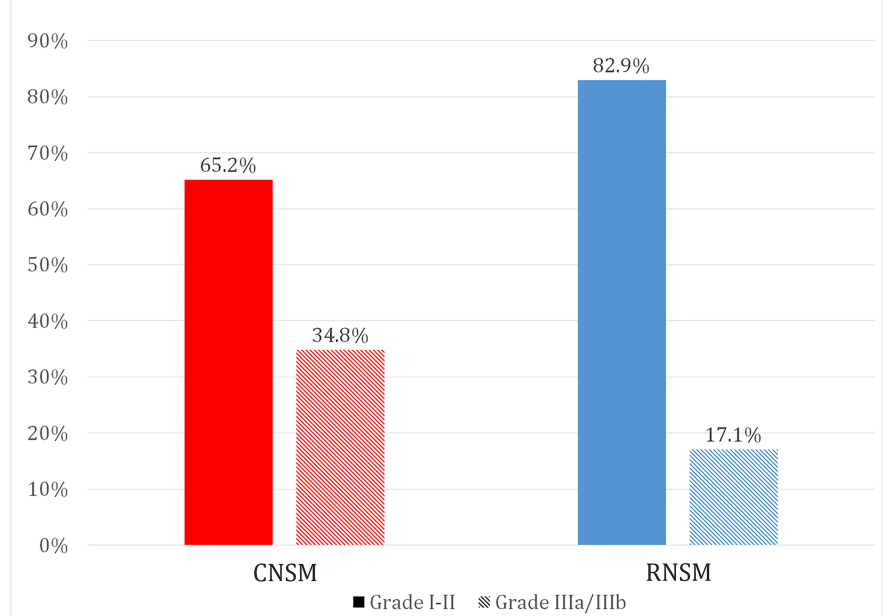

C

Rate of nipple necrosis $(p=0.026)$

$$
\begin{array}{r}
100 \% \\
90 \% \\
80 \% \\
70 \% \\
60 \% \\
50 \% \\
40 \% \\
30 \% \\
20 \% \\
10 \% \\
0 \%
\end{array}
$$

$15.2 \%$

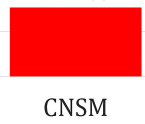

$2.4 \%$

RNSM

FIGURE 3 | Comparison of post-operative complications between conventional nipple-sparing mastectomy and robot-assisted nipple sparing mastectomy. (A) Rate of complications, (B) Grade of complications, (C) Rate of nipple necrosis. 
TABLE 3 | Multivariate analysis for risk factors related with high-grade complications.

\begin{tabular}{lrr}
\hline & \multicolumn{1}{c}{ Clavien-Dindo Classification $\geq$ Grade III } \\
\cline { 2 - 3 } & \multicolumn{1}{c}{ OR (95\% Cl) } & p-value \\
\hline Age $(\leq 50$ vs. $>50)$ & $0.751(0.381-1.480)$ & 0.408 \\
Breast volume $(\leq 310 \mathrm{~g}$ vs. $>310 \mathrm{~g})$ & $1.638(0.862-3.111)$ & 0.132 \\
Ptosis (Normal vs. Ptotic) & $0.904(0.489-1.673)$ & 0.748 \\
Operation time (min) & $1.005(1.004-1.007)$ & $<0.001$ \\
Operation method (CNSM vs. RNSM) & $0.406(0.167-0.986)$ & 0.046 \\
\hline
\end{tabular}

CNSM, conventional nipple-sparing mastectomy; RNSM, robot-assisted nipple-sparing mastectomy.

study presented that a transaxillary incision could be the incision of choice for NSM with valid, oncological safe, and excellent cosmetic results in breast cancer patients or $B R C A$ mutation carriers (31). For this reason, small axillary or lateral incisions in RNSM may have beneficial effects on the integrity of overlying skin and the NAC.

The rate of complications was not statistically different between the RNSM and the CNSM groups. Grades of post-operative complications were significantly different between the two groups. Compared to CNSM, RNSM showed lower rates of high-grade complications in the univariate and multivariate analysis. This different rate of high-grade complication may be due to different types of immediate reconstruction procedures. A previous study in our institution reported that reconstruction with TRAM free flap, LD flap with implant, and DTI presented with more post-operative NAC necrosis than reconstruction with a T/E (1). Similarly, another study reported that higher grades of post-operative complications occurred more commonly in patients with autologous reconstructions compared to those with implant-based reconstructions (32). In the present study, approximately one third $(27.7 \%)$ of patients in the CNSM group underwent autologous reconstructions, and this may influence the higher grade of post-operative complications in this group. Therefore, it is important to consider types of reconstruction procedure as a stratification factor when conducting randomized clinical trials in the future.

In the present study, RNSM was mainly performed on patients with small- to medium-sized breasts without ptosis. This is concordant with previous studies (19). Toesca et al. mainly enrolled women with small- to medium-sized breasts with low grade ptosis in their randomized clinical trial $(19,33)$. This may be due to the fact that implant-based reconstruction is suitable for small- to medium-sized breasts with low grade ptosis. Implantbased reconstructions constituted the major reconstruction method after RNSM because LD or TRAM flap requires additional incisions compared to implant-based reconstruction. Autologous reconstruction after RNSM remained as a technical challenge of robotic surgery.

Operation times for mastectomy were longer in the RNSM group than in the CNSM group in this study. Robotic surgery, including thyroidectomy, colectomy, and gastrectomy, presented with longer operation times than conventional surgery (34-36).

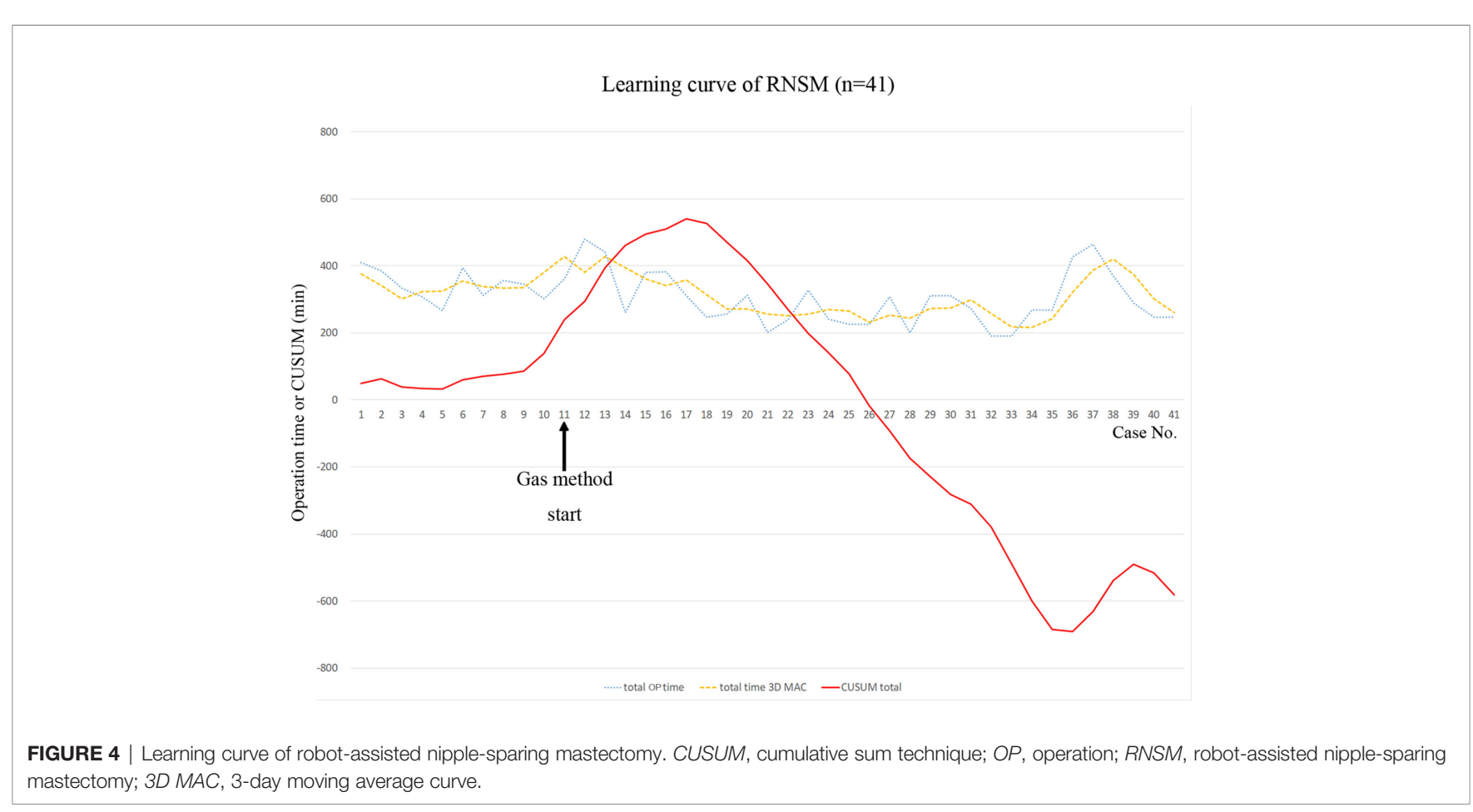


This is due to the development of the working space, the robot docking, and surgeon's experience (37). This is also the case with RNSM. Mean mastectomy time in the RNSM group was $181.5 \mathrm{~min}$, and it was longer than mastectomy time in the CNSM group (95.5 $\mathrm{min}$ ). However, as RNSM is a new technique, there was a learning period in the initial cases in this study. Even though operation times during RNSM decreased over time (Figure 4), a significant learning curve associated with a new technique such as RNSM may account for longer operation times compared to conventional procedures. Despite increased duration of mastectomy, console time in RNSM was approximately 1 hour (Table 2). Further studies regarding learning curves are necessary for comparisons of the two groups in terms of duration of operation.

Hospital stays were longer in the RNSM group than in the CNSM group. However, with a difference of only two days, there was no significant impact on clinical outcomes because there are differences in hospital stays according to surgeons' preferences (data not shown).

There are several limitations to this study. The retrospective design of this study may have led to selection bias. Propensity matching would be an alternative method to reduce the limitations of a retrospective study. Also, the numeric disparity between the RNSM and CNSM groups was another limitation. Patient satisfaction and cosmetic outcomes, which may be one of the main advantages of RNSM, were not measured. A lack of detailed information on reconstructive techniques, such as subpectoral or prepectoral techniques, was another limitation of the study. Oncologic outcomes, such as loco-regional recurrence-free survival, disease-free survival, and overall survival, are important end-points in the treatment of patients with breast cancer. Prospective studies with longer follow-ups are needed to overcome these limitations. However, to the best of our knowledge, this is the largest study to evaluate differences in terms of grades of complications and rates of nipple necrosis between RNSM and CNSM. Moreover, the results of the current study support the feasibility and safety of robotic mastectomy as a treatment option for women with breast cancer or $B R C A$ mutations.

\section{REFERENCES}

1. Ahn SJ, Woo TY, Lee DW, Lew DH, Song SY. Nipple-areolar complex ischemia and necrosis in nipple-sparing mastectomy. Eur J Surg Oncol (2018) 44(8):1170-6. doi: 10.1016/j.ejso.2018.05.006

2. Carlson GW, Chu CK, Moyer HR, Duggal C, Losken A. Predictors of nipple ischemia after nipple sparing mastectomy. Breast J (2014) 20(1):69-73. doi: $10.1111 /$ tbj.12208

3. Algaithy ZK, Petit JY, Lohsiriwat V, Maisonneuve P, Rey PC, Baros N, et al. Nipple sparing mastectomy: can we predict the factors predisposing to necrosis? Eur J Surg Oncol (2012) 38(2):125-9. doi: 10.1016/j.ejso.2011.10.007

4. Galimberti V, Morigi C, Bagnardi V, Corso G, Vicini E, Fontana SKR, et al. Oncological Outcomes of Nipple-Sparing Mastectomy: A Single-Center Experience of 1989 Patients. Ann Surg Oncol (2018) 25(13):3849-57. doi: 10.1245/s10434-018-6759-0

5. Gerber B, Krause A, Reimer T, Muller H, Kuchenmeister I, Makovitzky J, et al. Skin-sparing mastectomy with conservation of the nipple-areola complex and autologous reconstruction is an oncologically safe procedure. Ann Surg (2003) 238(1):120-7. doi: 10.1097/01.SLA.0000077922.38307.cd

\section{CONCLUSION}

This study indicated that RNSM may have some advantages in terms of lower nipple necrosis and grade of post-operative complications. Further multicenter studies evaluating the clinical implications of RNSM should be conducted in the future.

\section{DATA AVAILABILITY STATEMENT}

The original contributions presented in the study are included in the article/supplementary material. Further inquiries can be directed to the corresponding author.

\section{ETHICS STATEMENT}

The studies involving human participants were reviewed and approved by the institutional review board at Severance Hospital (4-2019-0510). Written informed consent for participation was not required for this study in accordance with the national legislation and the institutional requirements.

\section{AUTHOR CONTRIBUTIONS}

HSP, a principal investigator, conceived the ideas of the study. JL and HSP wrote the manuscript, and conducted the data analysis and interpretation. All authors contributed to the article and approved the submitted version.

\section{ACKNOWLEDGMENTS}

The authors would like to thank Dong-Su Jang, MFA (Medical Illustrator), for his help with illustrations. The result of this study was presented in 2019 Asian Congress of Robotic and Laparoscopic Surgery, Singapore.

6. Bishop CC, Singh S, Nash AG. Mastectomy and breast reconstruction preserving the nipple. Ann R Coll Surg Engl (1990) 72(2):87-9.

7. Sacchini V, Pinotti JA, Barros AC, Luini A, Pluchinotta A, Pinotti M, et al. Nipplesparing mastectomy for breast cancer and risk reduction: oncologic or technica problem? J Am Coll Surg (2006) 203(5):704-14. doi: 10.1016/j.jamcollsurg.2006.07.015

8. de Alcantara Filho P, Capko D, Barry JM, Morrow M, Pusic A, Sacchini VS. Nipple-sparing mastectomy for breast cancer and risk-reducing surgery: the Memorial Sloan-Kettering Cancer Center experience. Ann Surg Oncol (2011) 18(11):3117-22. doi: 10.1245/s10434-011-1974-y

9. Psaila A, Pozzi M, Barone Adesi L, Varanese A, Costantini M, Gullo P, et al. Nipple sparing mastectomy with immediate breast reconstruction: a short term analysis of our experience. J Exp Clin Cancer Res (2006) 25(3):309-12.

10. Lohsiriwat V, Rotmensz N, Botteri E, Intra M, Veronesi P, Martella S, et al. Do clinicopathological features of the cancer patient relate with nipple areolar complex necrosis in nipple-sparing mastectomy? Ann Surg Oncol (2013) 20 (3):990-6. doi: 10.1245/s10434-012-2677-8

11. Larson DL, Basir Z, Bruce T. Is oncologic safety compatible with a predictably viable mastectomy skin flap? Plast Reconstr Surg (2011) 127(1):27-33. doi: 10.1097/PRS.0b013e3181f9589a 
12. Rusby JE, Smith BL, Gui GP. Nipple-sparing mastectomy. Br J Surg (2010) 97 (3):305-16. doi: 10.1002/bjs.6970

13. Petit JY, Veronesi U, Orecchia R, Rey P, Didier F, Giraldo A, et al. The nipplesparing mastectomy: early results of a feasibility study of a new application of perioperative radiotherapy (ELIOT) in the treatment of breast cancer when mastectomy is indicated. Tumori (2003) 89(3):288-91. doi: 10.1177/ 030089160308900311

14. Daar DA, Abdou SA, Rosario L, Rifkin WJ, Santos PJ, Wirth GA, et al. Is There a Preferred Incision Location for Nipple-Sparing Mastectomy? A Systematic Review and Meta-Analysis. Plast Reconstr Surg (2019) 143 (5):906e-19e. doi: 10.1097/prs.0000000000005502

15. Radovanovic Z, Ranisavljevic M, Radovanovic D, Vicko F, Ivkovic-Kapicl T, Solajic N. Nipple-Sparing Mastectomy with Primary Implant Reconstruction: Surgical and Oncological Outcome of 435 Breast Cancer Patients. Breast Care (Basel) (2018) 13(5):373-8. doi: 10.1159/000489317

16. Huang Z, Chen K, Zhu L, Zeng J, Zhou Z, Li S. [Effectiveness of endoscopic nipple-sparing mastectomy combined with immediate breast reconstruction via axillary incision]. Zhongguo Xiu Fu Chong Jian Wai Ke Za Zhi (2018) 32 (11):1458-62. doi: 10.7507/1002-1892.201804027

17. Lai HW, Chen ST, Lin SL, Chen CJ, Lin YL, Pai SH, et al. Robotic NippleSparing Mastectomy and Immediate Breast Reconstruction with Gel Implant: Technique, Preliminary Results and Patient-Reported Cosmetic Outcome. Ann Surg Oncol (2019) 26(1):42-52. doi: 10.1245/s10434-018-6704-2

18. Sarfati B, Struk S, Leymarie N, Honart JF, Alkhashnam H, Tran de Fremicourt K, et al. Robotic Prophylactic Nipple-Sparing Mastectomy with Immediate Prosthetic Breast Reconstruction: A Prospective Study. Ann Surg Oncol (2018) 25(9):2579-86. doi: 10.1245/s10434-018-6555-x

19. Toesca A, Peradze N, Manconi A, Galimberti V, Intra M, Colleoni M, et al. Robotic nipple-sparing mastectomy for the treatment of breast cancer: Feasibility and safety study. Breast (2017) 31:51-6. doi: 10.1016/ j.breast.2016.10.009

20. Winter R, Haug I, Lebo P, Grohmann M, Reischies FMJ, Cambiaso-Daniel J, et al. Standardizing the complication rate after breast reduction using the Clavien-Dindo classification. Surgery (2017) 161(5):1430-5. doi: 10.1016/ j.surg.2016.11.028

21. Park HS, Kim JH, Lee DW, Song SY, Park S, Kim SI, et al. Gasless RobotAssisted Nipple-Sparing Mastectomy: A Case Report. J Breast Cancer (2018) 21(3):334-8. doi: 10.4048/jbc.2018.21.e45

22. Iqbal B, Buch A. Hormone receptor (ER, PR, HER2/neu) status and proliferation index marker (Ki-67) in breast cancers: Their onco-pathological correlation, shortcomings and future trends. Med J Dr D Y Patil Univ (2016) 9(6):674-9. doi: 10.4103/0975-2870.194180

23. Varga Z, Tubbs RR, Moch H. Concomitant detection of HER2 protein and gene alterations by immunohistochemistry (IHC) and silver enhanced in situ hybridization (SISH) identifies HER2 positive breast cancer with and without gene amplification. PloS One (2014) 9(8):e105961. doi: 10.1371/journal. pone. 0105961

24. Cheang MC, Chia SK, Voduc D, Gao D, Leung S, Snider J, et al. Ki67 index, HER2 status, and prognosis of patients with luminal B breast cancer. J Natl Cancer Inst (2009) 101(10):736-50. doi: 10.1093/jnci/djp082

25. Society KBC. 2019 The 8th Korean Clinical Practice Guideline for Breast Cancer. 8th ed. Seoul, Korea: Korean Breast Cancer Society (2019).

26. Park HS, Jeon CW. Learning curve for breast mass excision using a vacuumassisted biopsy system. Minim Invasive Ther Allied Technol (2014) 23(4):23540. doi: 10.3109/13645706.2014.894918

27. Bege T, Lelong B, Esterni B, Turrini O, Guiramand J, Francon D, et al. The learning curve for the laparoscopic approach to conservative mesorectal excision for rectal cancer: lessons drawn from a single institution's experience. Ann Surg (2010) 251(2):249-53. doi: 10.1097/SLA.0b013e3181b7fdb0

28. Park EJ, Kim MS, Kim G, Kim CH, Hur H, Min BS, et al. Long-term oncologic outcomes of laparoscopic right hemicolectomy during the learning curve period: comparative study with cases after the learning curve period. Surg Laparosc Endosc Percutan Tech (2015) 25(1):52-8. doi: 10.1097/sle.000000000 0000016

29. Khoury T, Kumar PR, Li Z, Karabakhtsian RG, Sanati S, Chen X, et al. Lobular neoplasia detected in MRI-guided core biopsy carries a high risk for upgrade: a study of 63 cases from four different institutions. Mod Pathol (2016) 29(1):2533. doi: $10.1038 /$ modpathol.2015.128

30. van Deventer PV. The blood supply to the nipple-areola complex of the human mammary gland. Aesthetic Plast Surg (2004) 28(6):393-8. doi: 10.1007/s00266-003-7113-9

31. Visconti G, Franceschini G, Bianchi A, Barone-Adesi L, Garganese G, Masetti R, et al. Transaxillary Nipple-Sparing Mastectomy and Direct-to-Implant Breast Reconstruction Using a Simplified Endoscopic Approach: Indications, Cosmetic Outcomes and Technical Refinements. Aesthetic Plast Surg (2020) 44(5):146675. doi: $10.1007 / \mathrm{s} 00266-020-01792-1$

32. Wilkins EG, Hamill JB, Kim HM, Kim JY, Greco RJ, Qi J, et al. Complications in Postmastectomy Breast Reconstruction: One-year Outcomes of the Mastectomy Reconstruction Outcomes Consortium (MROC) Study. Ann Surg (2018) 267(1):164-70. doi: 10.1097/sla.0000000000002033

33. Toesca A, Invento A, Massari G, Girardi A, Peradze N, Lissidini G, et al. Update on the Feasibility and Progress on Robotic Breast Surgery. Ann Surg Oncol (2019) 26(10):3046-51. doi: 10.1245/s10434-019-07590-7

34. Pan JH, Zhou H, Zhao XX, Ding H, Wei L, Qin L, et al. Robotic thyroidectomy versus conventional open thyroidectomy for thyroid cancer: a systematic review and meta-analysis. Surg Endosc (2017) 31(10):3985-4001. doi: 10.1007/ s00464-017-5433-0

35. Benlice C, Aytac E, Costedio M, Kessler H, Abbas MA, Remzi FH, et al. Robotic, laparoscopic, and open colectomy: a case-matched comparison from the ACS-NSQIP. Int J Med Robot (2017) 13(3):e1783. doi: 10.1002/rcs.1783

36. Solaini L, Bazzocchi F, Pellegrini S, Avanzolini A, Perenze B, Curti R, et al. Robotic vs open gastrectomy for gastric cancer: a propensity score-matched analysis on short and long-term outcomes. Int J Med Robot (2019) 15(5): e2019. doi: 10.1002/rcs.2019

37. Kang SW, Lee SH, Ryu HR, Lee KY, Jeong JJ, Nam KH, et al. Initial experience with robot-assisted modified radical neck dissection for the management of thyroid carcinoma with lateral neck node metastasis. Surgery (2010) 148 (6):1214-21. doi: 10.1016/j.surg.2010.09.016

Conflict of Interest: HP received honoraria including consulting fee and travel support from Intuitive Surgical Korea and a research grant from Intuitive Surgical, Inc. which were not related with the data and analysis of the current study.

The remaining authors declare that the research was conducted in the absence of any commercial or financial relationships that could be construed as a potential conflict of interest.

Copyright $\odot 2021$ Lee, Park, Lee, Lee, Song, Lew, Kim, Park and Kim. This is an openaccess article distributed under the terms of the Creative Commons Attribution License (CC BY). The use, distribution or reproduction in other forums is permitted, provided the original author(s) and the copyright owner(s) are credited and that the original publication in this journal is cited, in accordance with accepted academic practice. No use, distribution or reproduction is permitted which does not comply with these terms. 